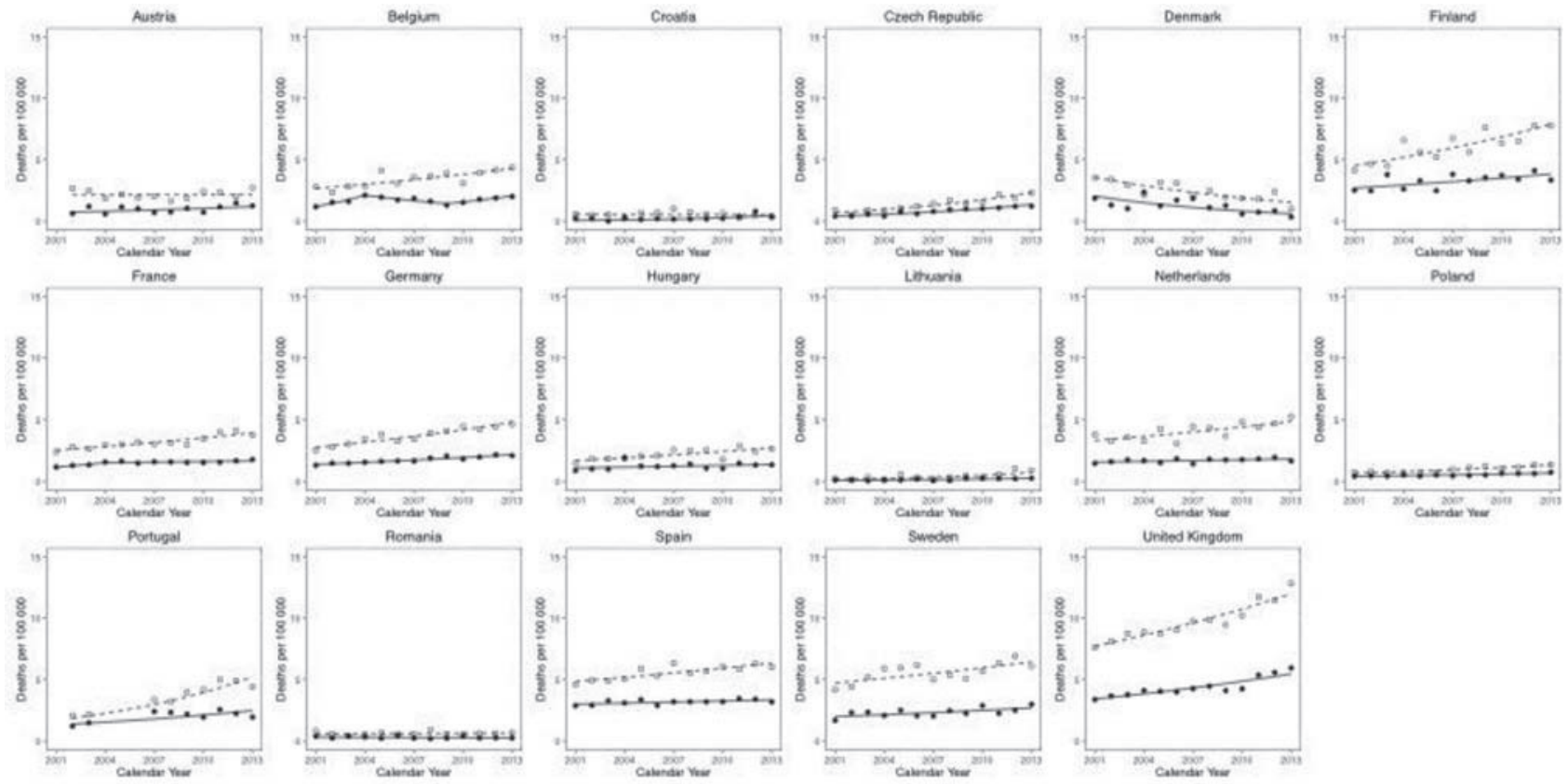

Abstract S124 Figure 1 Idiopathic pulmonary fibrosis mortality trends of male and females in 17 European countries. Lines represent result of Joinpoint analyses dashed and continuous lines for males and females, respectively. Squares (males) and circles (females) represent raw data, where symbol is absent data has been imputed for respective year.

observed in the UK and Finland. Rates were also substantially higher in males with gender disparity increasing across the period.

Interpretation Reported IPF mortality appears to be increasing across the EU, however there is substantial variation in mortality trends and overall reported mortality rates between countries. There are likely to be differences in coding practices and reporting levels between countries, particularly with specialist knowledge and equipment required to diagnose IPF.

\section{S125 DEATHS FROM RESPIRATORY DISEASE IN THE UK COMPARED TO EU15+ COUNTRIES: AN OBSERVATIONAL ANALYSIS OF NATIONAL MORTALITY STATISTICS, 1985 - 2013}

${ }^{1} \mathrm{JD}$ Salciccioli, ${ }^{2} \mathrm{DC}$ Marshall, ${ }^{3} \mathrm{M}$ Maruthappu, ${ }^{4} \mathrm{~J}$ Shalhoub. 'Mount Auburn Hospital, Cambridge, US; ${ }^{2} J o h n$ Raddliffe Hospital, Oxford, UK; ${ }^{3}$ University College London, London, UK; ${ }^{4}$ Imperial College London, London, UK

\subsection{6/thoraxjnl-2017-210983.131}

Introduction Respiratory disease consistently ranks among the most fatal disease processes globally. Previous reports from Global Burden of Disease have identified higher burden of respiratory disease in the UK compared to similar health systems.

Methods We compared UK to EU15 + countries (i.e., Austria, Belgium, Denmark, Finland, France, Germany, Greece, Ireland, Italy, Luxembourg, Netherlands, Portugal, Spain, and Sweden, Australia, Canada, United States, and Norway) because of similar or higher health expenditure in these countries. We obtained respiratory-related, sex-specific mortality data from countries of interest from the WHO World Mortality database between 1985 and 2013 and covariate data from the World Bank's Development Indicators DataBank. Age-standardised death rates (ASDR) were computed using the WHO World standard population. We used Joinpoint regression analysis to test changes in trends for respiratory disease. We used Poisson regression to test the difference between UK and EU15 + countries after controlling for smoking and pollution factors.

Results In the UK, there was a significant decreasing trend in respiratory-related mortality between 1985 and 1991 with an estimated annual percentage change (EAPC) of -0.89 and -1.70 , for men and women respectively. Between 1994 and 2013, there was a steady decline in ASDR with EAPC -2.06 and -0.85 , for men and women, respectively. For EU15 + men, there was a decreasing trend in ASDR between until 1999 with EAPC -0.81 and from 1999 onwards the EAPC was -2.14. For EU15 + women, there was an increasing trend in ASDR until 2002 with EAPC of +1.48 which was followed by overall decreasing trend with EAPC -0.44 until 2013. After multivariable adjustment for pollution exposure and smoking prevalence in each country there was a persistent significant difference in ASDR with approximately 20\% higher mortality in UK compared to EU15 + $(p=0.009)$.

Conclusion There was significantly greater mortality from respiratory-related illnesses in UK compared to EU15 +over the period from 1985 to 2013 after controlling for smoking and pollution exposure. System-level and population-level factors may contribute to this difference and additional investigations are necessary to further explain these differences.

\section{REFERENCE}

1. Murray et al. Lancet 2013;997. 